\title{
A Influência da Fisioterapia Aquática na Função e Equilíbrio no Acidente Vascular Cerebral
}

\author{
Aquatic Physiotherapy Influence In Function and Balance In Stroke
}

\author{
Cristiane Helita Zorél Meneghetti ${ }^{1}$, Lucas Carraro ${ }^{2}$, Leandro Augusto \\ Leonello ${ }^{2}$, Ana Carolina Texeira Batistella ${ }^{3}$, Luiz Carlos Ferracini Júnior ${ }^{4}$
}

\section{RESUMO}

Objetivo. Verificar a influência da fisioterapia aquática na independência funcional e equilíbrio no Acidente Vascular Cerebral (AVC). Método. Foi realizado um estudo de caso com um indivíduo acometido por AVC, gênero feminino, 36 anos de idade com diagnóstico disfuncional de hemiparesia à esquerda. $\mathrm{O}$ instrumento utilizado para avaliar o equilíbrio foi à escala de equilíbrio funcional de Berg (EEFB - versão brasileira) composta por quatorze tarefas, já para avaliar a funcionalidade foi utilizada a escala de medida de independência funcional (MIF - versão brasileira) composta por dezoito itens. A fisioterapia aquática consistiu em duas sessões semanais de cinquenta minutos num período de três meses, totalizando 24 sessóes, empregando o método Halliwick. Resultados. Na avaliação após a intervenção, foi observada uma melhora na pontuação na escala de EEFB de 15 para 40 pontos, da mesma forma na escala da MIF verificou-se um aumento da pontuação de 91 para 108 pontos. Conclusáo. O programa de fisioterapia aquática proporcionou a participante com AVC, uma melhora no equilíbrio e na funcionalidade.

Unitermos. Acidente Vascular Encefálico, Fisioterapia, Equilíbrio, Funcionalidade.

Citaçáo. Meneghetti CHZ, Carraro L, Leonello LA, Batistella ACT, Ferracini Júnior LC. A Influência da Fisioterapia Aquática na Função e Equilíbrio no Acidente Vascular Cerebral.

\begin{abstract}
Objective. Verify the influence of aquatic physiotherapy in functional independence and balance in stroke. Method. It was made a study of a case of a patient with stroke, female, 36 years old, with dysfunctional diagnosis of left-side hemiparesis. The instrument used to assess the balance was the Berg Balance Scale (BBC) composed by fourteen tasks and to assess functionality, the Functional Independence Measure (FIM) scale, composed by eighteen items, was used. Aquatic physiotherapy consisted of two weekly sessions of fifty minutes in a three month period of time, doing in total 24 sessions, using Halliwick method. Results. In the assessment after the intervention, an improvement was observed in the BBC scale from 15 to 40 points, also in the FIM scale an improvement from 91 to 108 points was verified. Conclusion. The aquatic physiotherapy program provided the stroke patient an improvement in balance and functionality.
\end{abstract}

Keywords. Stroke, Physical Therapy, Balance, Functioning.

Citation. Meneghetti CHZ, Carraro L, Leonello LA, Batistella ACT, Ferracini Júnior LC. Aquatic Physiotherapy Influence In Function and Balance In Stroke.
Trabalho Realizado no Centro Universitário Hermínio Ometto - UNIARARAS, Araras-SP, Brasil.

1.Fisioterapeuta, Mestre em Distúrbios do Desenvolvimento - MACKENZIE, São Paulo-SP, Brasil.

2.Fisioterapeuta, Centro Universitário Hermínio Ometto - UNIARARAS, Araras-SP, Brasil.

3.Fisioterapeuta, Mestre em Fisioterapia - UNIMEP, Piracicaba-SP, Brasil

4.Fisioterapeuta, Mestrando em Ciências Biomédicas - UNIARARAS, ArarasSP, Brasil.
Endereço para correspondência: Cristiane Helita Zorél Meneghetti

Rua das Nogueiras, 95, Jardim Nova Araras CEP 13601291, Araras-SP, Brasil.

E-mail: crismeneghetti@yahoo.com.br

Relato de Caso Recebido em: 16/09/11 Aceito em: 21/02/12 Conflito de interesses: não 


\section{INTRODUÇÃO}

O Acidente Vascular Cerebral (AVC) é uma interrupção súbita do fluxo cerebral vascular, resultando em lesôes celulares e danos às funçôes neurológicas, podendo ocorrer por tipo isquêmico ou hemorrágico ${ }^{1-3}$. As consequências acarretadas pelo AVC ocasionam danos físicos como plegias ou paresias de um ou ambos os membros, alteraçóes sensoriais e espasticidade; danos psicoafetivos como quadros depressivos, ansiedade e agressividade; danos cognitivos como problemas de memória, atenção e concentração, alteração da linguagem e funçôes executivas, dificuldades no planejamento de açóes e déficit perceptual e perda do mecanismo de controle postural ${ }^{4-6}$.

O controle postural possui dois objetivos comportamentais: a orientação e o equilíbrio postural. A orientação postural está relacionada ao posicionamento e ao alinhamento dos segmentos corporais em relação aos outros e em relação ao ambiente. O equilíbrio postural é o estado em que todas as forças que atuam sobre o corpo estão balanceadas para manter o corpo na posição e orientaçáo desejada ${ }^{7}$.

As alteraçóes no controle postural provocadas pelo AVC geram limitaçôes nas atividades funcionais e uma das formas de recuperação é baseada na intervenção pela fisioterapia aquática ${ }^{8,9}$.

A fisioterapia aquática tem como objetivo promover o máximo de independência funcional possível ao paciente, minimizando as respostas anormais e potencializando os movimentos apropriados, beneficiando-se dos princípios físicos e termodinâmicos da água. Entre as quais se destacam o empuxo, a pressão hidrostática e viscosidade $^{10}$.

Dentre os métodos utilizados na fisioterapia aquática tem-se o método Halliwick, que prioriza a prática de atividades aquáticas, sendo também empregado para intervenção terapêutica. É uma técnica que segue um princípio de desprendimento. Apresenta as seguintes posturas: bastão vertical (o corpo mantido na posição ereta) e bastão horizontal (o corpo mantido na posição horizontal onde é facilmente rodado em torno de seu eixo longitudinal), bola (definida como posição "enrolada”, fornecendo equilíbrio mais estável e considerável esforço é necessário para alterar a posição do corpo) e por fim, posição de cubo (definida como se o indivíduo estivesse sentado na água) $)^{9,10}$.
Desta forma, o objetivo do estudo foi verificar a influência da fisioterapia aquática na independência funcional e equilíbrio no Acidente Vascular Cerebral (AVC).

\section{MÉTODO}

\section{Amostra}

Tratou-se de um modelo de estudo de caso, que obteve aprovação do Comitê de Ética e Mérito em Pesquisa sob o parecer $n^{\circ} 811 / 2008$ do Centro Universitário Hermínio Ometto - UNIARARAS.

Participou do estudo um indivíduo com AVC, gênero feminino, 36 anos de idade, recrutado na Clínica Escola de Fisioterapia - UNIARARAS, com diagnóstico disfuncional de hemiparesia à esquerda desproporcional com predomínio crural. Antes de iniciar a avaliação, o paciente assinou o termo de consentimento livre e esclarecido.

Para a realização da avaliação do equilíbrio o instrumento utilizado foi a Escala de Equilíbrio Funcional de Berg (EEFB) - versão brasileira ${ }^{11}$, composto por catorze questóes, onde as pontuaçóes dos subitens variam de 0 a 4 pontos.

O zero significa que o participante é incapaz de realizar a tarefa pretendida e o quatro refere-se ao participante que executa os movimentos solicitados, de forma independente e permanece numa determinada posição durante todo ou quase o tempo previsto para aquela tarefa.

\section{Procedimentos}

Este teste é constituído por uma escala de 14 tarefas comuns que envolvem o equilíbrio estático e dinâmico tais como: alcançar, girar, transferir-se, permanecer em pé e levantar-se. A realização das tarefas é avaliada através de observação e a pontuação se totaliza no máximo de 56 pontos. Estes pontos devem ser subtraídos caso o tempo ou à distância não seja atingido, se o participante necessite de supervisão para a execução da tarefa ou, se o participante apóia-se num suporte externo ou recebe ajuda do examinador.

$\mathrm{Na}$ amplitude de 56 a 54, cada ponto a menos é associada a um aumento de 3 a $4 \%$ abaixo no risco de quedas, de 54 a 46 a alteraçáo de um ponto é associada a um aumento de 6 a $8 \%$ de chances, sendo que abaixo de 36 pontos o risco de quedas é quase $100 \%$.

Já para a avaliação da funcionalidade o instrumen- 
to utilizado foi a Escala de Medida de Independência Funcional (MIF) que avalia 18 categorias pontuadas de um a sete e classificadas quanto ao nível de dependência para a realização da tarefa. As categorias são agrupadas em seis dimensóes: MIF motora - autocuidados (alimentação, higiene pessoal, banho, vestir metade superior, vestir metade inferior, utilização do vaso sanitário), controle de esfíncteres (controle da diurese e defecação), transferências (leito, cadeira, cadeira de rodas, vaso sanitário, banheiro, chuveiro), locomoção (marcha, cadeira de rodas, escadas). MIF cognitiva - comunicação (compreensão, expressão) e cognição social (interação social, resolução de problemas, memória). Somando-se os pontos das dimensōes da MIF, obtém-se um escore total mínimo de 18 e o máximo de 126 pontos, que caracterizam os níveis de dependência pelos subescores ${ }^{12}$.

Para cada item atribui-se uma nota que varia de 1 a 7 de acordo com o grau de dependência sendo 7 independência completa; 6- independência modificada; 5- supervisão; 4- ajuda mínima (indivíduo realiza 75\% da tarefa); 3- ajuda moderada (indivíduo realiza 50\% da tarefa); 2- ajuda máxima (indivíduo realiza $25 \%$ da tarefa), 1- ajuda total.

A MIF total pode ser dividida em quatro subescores, de acordo com a pontuação total obtida: a) 18 pontos: dependência completa (assistência total); b) 19-60 pontos: dependência modificada (assistência de até 50\% da tarefa); c) 61-103 pontos: dependência modificada (assistência de até 25\% da tarefa); d) 104-126 pontos: independência completa ${ }^{13}$.

Após a avaliação iniciou-se a intervenção de fisioterapia aquática, utilizando o método Halliwick. As sessóes foram realizadas na piscina terapêutica da Clínica Escola de Fisioterapia da Uniararas, com dimensóes de 4,5 m de largura por $9 \mathrm{~m}$ de comprimento, profundidade de $80 \mathrm{~cm}$ a 1 metro, com temperatura variando entre $28^{\circ}$ e $33^{\circ} \mathrm{C}$. Ao total foram realizadas 24 sessóes de 50 minutos cada, duas vezes por semana, por um período de 3 meses.

No método Halliwik foram realizados exercícios de fortalecimento para os membros superiores utilizando flutuadores e exercícios de fortalecimento em cadeia cinética fechada para membros inferiores na posição de cubo com água ao nível dos acrômios; exercícios para rea- çóes de equilíbrio e propriocepção utilizando aquatube $e^{\oplus}$ prancha, exercícios de fortalecimento para membros inferiores utilizando tornozeleiras de um quilograma na postura de bastão vertical e na postura de bastão horizontal em prono. Destinaram-se as três primeiras sessóes para a adaptação do participante ao meio líquido, familiarizando-o com a profundidade, a temperatura e a flutuação em meio aquático, com mínima ajuda do terapeuta.

Após o programa de fisioterapia aquática a participante foi novamente submetida à avaliação do equilíbrio e da funcionalidade.

\section{RESULTADOS}

Antes da intervenção da fisioterapia aquática, o indivíduo apresentava uma pontuação pela EEFB de 15 pontos, mostrando um risco de queda de $100 \%$. Após a intervenção a pontuação na EEFB foi de 40 pontos.

Da mesma forma, em relação à MIF total, constatou-se uma melhora na funcionalidade após o programa de fisioterapia aquática passando de 91 pontos para 108 pontos. Já a média da pontuação da MIF motora antes da intervenção foi de 5,5 1,0 mostrando que o indivíduo precisava de supervisão para realização das atividades funcionais e, que após a intervenção a média da pontu-

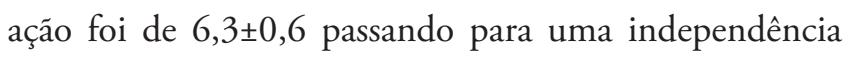

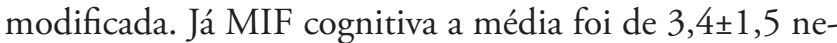
cessitando de uma ajuda moderada e após o programa de fisioterapia aquática passou para 4,7 $\pm 1,0$ apresentando uma ajuda mínima.

\section{DISCUSSÃO}

De maneira geral, verificou-se que após a intervenção da fisioterapia aquática, a participante apresentou melhora no equilíbrio e na funcionalidade.

Os instrumentos utilizados para este estudo foram a EEFB e a MIF. A EEFB é uma escala prática, simples, precisa e confiável que tem como objetivo mensurar o equilíbrio e assim nortear o tratamento, como evidenciado em vários estudos ${ }^{11,14,15}$. Da mesma forma, a MIF é provavelmente o mais amplo instrumento para mensurar a capacidade funcional, sendo um indicador importante na rotina clínica por quantificar a independência na realização das atividades de vida diária (AVD's) ${ }^{12,13,16}$. 
Diversos estudos confirmam sua confiabilidade e eficácia clínica atendendo a critérios de precisão, praticidade e facilidade ${ }^{16,17}$.

Para alguns autores, o fato dos indivíduos com AVC apresentarem alteraçôes no controle motor, no sistema somatossensorial, no perceptivo, no cognitivo e no mecanismo de controle postural, faz com que os pacientes se empenhem em buscar o centro de equilíbrio, uma vez que este potencializa a realização das AVD's ${ }^{18-20}$.

Para nos orientarmos, necessitamos do sistema somatossensorial, que provém informação sobre as cargas e as posiçôes relativas das partes do corpo, do sistema visual, que fornece informação sobre movimento e indícios para julgamento da verticalidade, mantendo a horizontalidade do olhar, e por fim do sistema vestibular, que nos informa a posição da cabeça em relação à gravidade e sobre os movimentos da própria cabeça ${ }^{21,22}$.

As experiências provocadas pela água podem estimular a potencialidade plástica do sistema nervoso central por meio de estímulos sensitivos e motores, favorecendo um maior controle motor, reaçóes de equilíbrio além de promover o máximo de independência funcional ao paciente $\mathrm{e}^{9,23-25}$.

A água é utilizada há muito tempo para fins terapêuticos, e suas propriedades físicas auxiliam na recuperação e aquisição de novas habilidades. A turbulência desestabiliza o indivíduo gerando estímulos constantes para o ajuste postural, já a viscosidade, aumenta o tempo de queda na água, proporcionando assim, um tempo maior para respostas como as reaçôes de equilíbrio, endireitamento e proteção, o que estimula os mecanismos de sinergia postural, favorecendo assim a melhora na funcionalidade ${ }^{9,24,26}$.

O recrutamento de maior número de indivíduos permitirá confirmar estes resultados, de modo a poder afirmar a eficácia da fisioterapia aquática na melhora do equilíbrio e na funcionalidade no AVC.

\section{CONCLUSÃO}

Conclui-se que após a intervenção da fisioterapia aquática, a participante com AVC apresentou melhora no equilíbrio e na realização das atividades funcionais.

\section{REFERÊNCIAS}

1.Rietdyk S, Patla AE, Winter DA, Ishac MG, Litlle CE. Balance recovery from medio-lateral perturbations of the upper body during standing. J. Biomech 1999;32:49-1158.

http://dx.doi.org/10.1016/S0021-9290(99)00116-5

2.O'Sullivan SB, Schmitz TJ. Acidente vascular cerebral. In: O'Sullivan SB. Fisioterapia: avaliaçấo e tratamento. $2^{a}$ ed. São Paulo: Manole, 1993, p.385-421. 3.Moura EW, Silva PAC. Abordagem fisioterapêutica no acidente vascular encefálico. In: Neves RCM, Pires MA. Fisioterapia: aspectos clínicos e práticos da reabilitação. São Paulo: Artes Médicas, 2005, p.360-81.

4.Bobath B. Hemiplegia em Adultos. 3a ed. Manole, 2001, 209p.

5.Peluso AQL, Lima FMR. Eletroestimulação na espasticidade decorrente de acidente vascular encefálico. Rev fisioter Ser 2007; 02: 40-5.

6.Teixeira E, Sauron FN, Santos LSB, Oliveira MC. Acidente vascular encefálico. In: Ares MJJ. Terapia ocupacional na reabilitaçăo física. São Paulo: Roca, 2003, p.3-16.

7.Horak FB, Macpherson JM. Postural Orientation and equilibrium. In: Rowell LB, Sherpherd JT. (Ed.) Handbook of physiology. New York: Oxford University Press, 1996, p.255-92.

8.Sacchelli T, Accacio LMP, Radl ALM. Fisioterapia Aquática. Barueri: Manole, 2007, 350p.

9.Schanzer GS, Queiroz SS. Fisioterapia aquática aplicada à neurologia. In: Sacchelli T, Accacio LMP, Radl ALM. Fisioterapia aquática. Barueri: Manole; 2007. (Manuais de Fisioterapia). p.191-202.

10.Guazzelli ABA. Reabilitação aquática aplicada na lesāo medular. In: Jakaitis F, editor. Reabilitação e terapia aquática: aspectos clínicos e práticos. São Paulo: Roca; 2007. p.101-55.

11. Miyamoto ST, Lombardi Júnior I, Berg KO, Ramos LR, Natour J. Brazilian version of the Berg balance scale. Braz J Med Biol Res 2004;37:1411-21.

http://dx.doi.org/10.1590/S0100-879X2004000900017

12.Riberto M, Miyazaki MH, Filho DJ, Sakamoto H, Battistella LR. Reprodutibilidade da versâo brasileira da medida de independência funcional. Acta Fisiatr 2001;8:45-52.

13.Riberto M, Miyazaki MH, Jucá SSH, Sakamoto H, Pinto PPN, Battistella LR. Validação da versão brasileira da medida de independência funcional. Acta Fisiatr 2004;11(2):72-6.

14.Stevenson TJ. Detecting change in patients with stroke using the Berg Balance Scale. Aust J Physiother 2001;47:29-38.

15. Oliveira R, Cacho E, Walker A, Borges G. Post-stroke motor and functional evaluations: a clinical correlation using Fugl-Meyer assessment scale, Berg balance scale and Barthel index. Arq neuropsiquiatr 2006;64:731-5.

http://dx.doi.org/10.1590/S0004-282X2006000500006

16.Ricci NA, Kubota MT, Cordeiro RC. Concordância de observaçóes sobre a capacidade funcional de idosos em assistência domiciliar. Rev Saúde Pública 2005;39(4):655-62.

http://dx.doi.org/10.1590/S0034-89102005000400021

17.Torriani C, Mota EPO, Kazurayama SHP, Burin SR, Mengatti T, Caminho $\mathrm{J}$, et al. Relaçấo entre independência e o nível de disfunção motora e funcional em pacientes hemiparéticos. Rev Neurocienc 2007;15:32-6.

18.Umphred DA. Fisioterapia neurológica. $2^{\mathrm{a}}$ ed. São Paulo: Manole, 1994, 876p. 19.Davies PM. Passos a Seguir: um manual para o tratamento da hemiplegia no adulto. São Paulo: Manole, 1996, 314p.

20.Davies PM. Hemiplegia: tratamento para pacientes após AVC e outras lesôes cerebrais. 2a ed. Barueri: Manole, 2008, 636p.

21.Jeka J, Oie K, Kiemel KS. Multisensory information for human postural control: integrating touch and vision Exp Brain Res 2000;134:107-25.

http://dx.doi.org/10.1007/s002210000412 
22.Barela JA. Estratégias de controle em movimentos complexos: ciclo percepção-ação no controle postural. Rev paul Educ Fís 2000;3:79-88.

23.Ruoti RG, Morris DM, Cole AJ. Reabilitação Aquática. Sáo Paulo: Manole, 2000, 463p.

24.Jakaitis F. Reabilitação e terapia aquática: aspectos clínicos e práticos. São
Paulo: Roca, 2007, 282p.

25.Fiorelli A, Arca EA. Hidrocinesioterapia: princípios e técnicas terapêuticas. Bauru: EDUSC, 2002, 104p.

26.Campion MR. Hidroterapia: princípios e prática. São Paulo: Manole, 2000, 332p. 